\title{
Why Individual Behavior Is Key to the Spread of Viruses Such as Covid-19
}

\author{
Michael Grabinski, Galiya Klinkova \\ Department of Business and Economics, Neu-Ulm University, Wileystr, Neu-Ulm, Germany \\ Email: michael.grabinski@uni-neu-ulm.de,galiya.klinkova@uni-neu-ulm.de
}

How to cite this paper: Grabinski, M., \& Klinkova, G. (2020). Why Individual Behavior Is Key to the Spread of Viruses Such as Covid-19. Theoretical Economics Letters, 10, 299-304.

https://doi.org/10.4236/tel.2020.102020

Received: February 22, 2020

Accepted: April 20, 2020

Published: April 23, 2020

Copyright (อ 2020 by author(s) and Scientific Research Publishing Inc. This work is licensed under the Creative Commons Attribution International License (CC BY 4.0).

http://creativecommons.org/licenses/by/4.0/

\begin{abstract}
Diseases are spread due to the behavior of people. One may have millions of differently behaving people. For simplicity, humankind is only considering the average behavior. However, this simplification can lead to results tremendously different from the exact solution in at least some applications when non-linear differential equations are used. In this letter, we prove that the mistake is very big for the spread of infections like Covid-19. Ten percent ignoring the rules can almost ruin the extremely careful behavior of the remaining ninety percent. This is totally different to most business situations where considering eighty percent is sufficient ("80-20-rule"). This may explain why the spread of the Covid-19 pandemic and the impact of measures against it are hard to predict for decision makers.
\end{abstract}

\section{Keywords}

Covid-19, Disease Spread, Average, Non-Linear Differential Equations, Chaos

\section{Introduction}

Any growth rate, be it population growth or the spread of a virus infection, is exponential in the beginning. Because nothing is infinite, there will be some saturation. There is a variety of models which are very similar at least for the effects discussed here. They are also used to create charts like Figure 1. In this case, we see the development for the spread of Covid-19 over time. It can become narrow and high (all cases within a short time span) or wide and low (cases are spread over a long time). Though the total number of cases stays the same, the latter one is more desirable. Hospitals can handle the second scenario much better. Economically speaking it is easier to bear some losses over two years for example, rather than a catastrophe within three months. The obvious measures to obtain the second scenario are to ensure people to behave carefully. 


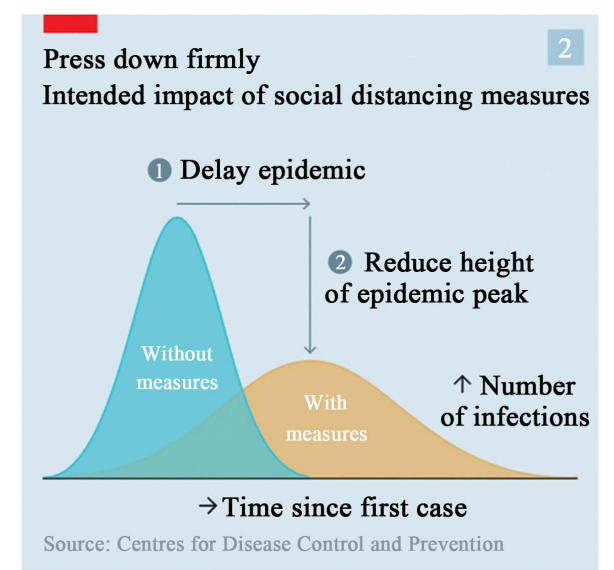

Figure 1. Development of desease (The Economist, 2020).

This letter does not intend to discuss the best measures. Neither will we calculate economic losses. We will point to the mathematics behind simulations. In order to get the curves of Figure 1 one has to solve a differential equation. As an input factor one has to make assumptions about how many people are infected within what time period, the basic reproduction number $R_{0}$. These assumptions lead to the different curves in Figure 1.

Because every human being behaves differently, we have a potential infection rate for each individual. Obviously, one cannot make an assumption about every citizen. Therefore, one considers an average instead. This is comparable to population growth, which depends on the birthrate per woman. There one also considers the average number of kids per woman (e.g. 1.3). Quite recently it has been proven by Grabinski \& Klinkova (2019) that doing so is only allowed in $I i$ near differential equations when aiming for the exact solution. For population growth, the mistake is not very big in most cases. This is because one considers either the starting point (pure exponential growth governed by a linear differential equation) or the saturation which is almost stationary. And without time dependence we cannot have the effect discussed here. Another example in Grabinski \& Klinkova (2019) is the diffusion model of marketing. Using an average diffusion constant as a simplification led to the conclusion that the diffusion model shows chaos (Weiber, 1993) which is incorrect. The finance world is also full of non-linear differential equations such as pricing by the Black Scholes differential Equation (Black \& Scholes, 1973). Though the mistake due to using average constants is most likely tremendous, the error is prohibitively difficult to estimate. This is due to the fact that these models are not correct in the first place and give a rough estimate only. For more details about it, see also (Grabinski \& Klinkova, 2019).

In the example of the spreading of diseases nobody doubts that the models are correct in terms of their structure. It is just hard to guess ex-ante what the input parameters are. An ex-post comparison between model and reality would lead to the effective parameters. In Chapter 2 we will introduce a simple model which 
produces curves like the one of Figure 1. However, the outcome is strikingly different whether one takes an average infection rate or a varying one. Assume that if everybody in the population behaved carefully, this would lead to a spread of the disease over e.g. 24 months, while if everybody behaved badly, the disease would spread over three months only, for instance. If we assume that half of the population behaves badly and the other half is extremely careful, it may be a quite reasonable behavior on average. This average would lead to a spread of the disease over 24 months if everybody shows this behavior. However, the actions of the badly behaving half will lead to a spread of the disease over little more than three months in total. Because the spread is exponential rather than linear, the extremely careful half will be infected by the badly behaving with almost no delay making their effort worthless.

In Chapter 3 we will draw conclusions. Instead of limiting the average number of social contacts in order to curb the spread of Covid-19, one has to make sure that almost everybody takes a minimum of caution.

\section{Growth Model}

As one gets from almost all mathematical textbooks (e.g. Bronshtein et al., 2007) a population $n(t)$ will grow proportionally to $n$ :

$$
\dot{n}(t)=\frac{1}{\tau} \cdot n(t)
$$

Equation (1) will lead to unlimited growth. The constant $1 / \tau$ is the speed. A small $\tau$ will lead to rapid growth, a big one to slow growth. In the case of the spread of diseases, $\tau$ can be estimated by the reproduction number $R_{0}$. But this is not our goal here. We are just assuming different values of $\tau$ for different parts of the population.

Of course, the unlimited growth of Equation (1) holds only in the beginning. In the case of Covid-19 experts reckon that about two thirds of the population will get infected eventually without vaccination. In order to stay general, we normalize the part of the population being infected eventually to 1 . For example for Germany, with roughly 80 million inhabitants and about 50 million potential infections, this means that in case of 1000 infected people $n$ takes a value of $2 \times 10^{-5}$. There are various approaches to put a limit on Equation (1). The simplest is a limitation borrowed from the logistic equation:

$$
\dot{n}(t)=\frac{1}{\tau} \cdot n(t) \cdot(1-n(t))
$$

This approach is not untypical for growth limitations. When $n(t)$ approaches its maximum 1, growth becomes slower and slower. The solution of Equation (2) can be given in a closed form:

$$
n(t)=\frac{n_{0} \cdot \mathrm{e}^{t / \tau}}{1-\left(1-n_{0}\right) \cdot \mathrm{e}^{t / \tau}}
$$

In order to get curves like the one of Figure 1 one has to take the derivative 
with respect to time of Equation (3):

$$
\dot{n}(t)=\frac{n_{0}}{\tau} \cdot \frac{\mathrm{e}^{t / \tau}\left(1-n_{0}\right)}{\left(1+\left(\mathrm{e}^{t / \tau}-1\right) n_{0}\right)^{2}}
$$

A presumably very similar formula has been used to produce the curves of Figure 1. We now plot Equation (4) for three different values of $\tau$. We always took $2 \times 10^{-5}$ infections as the starting value $n_{0}$. The time units are arbitrary. One may think of months. Then the first society (blue curve, tallest) has everybody infected within under one year, the second (orange curve) in about two years and the third (green curve, smallest) within over three years. The curves of Figure 2 are the results for three societies behaving differently. If half of the society behave like in the blue curve and the other half like in the green curve, one may naively conclude that the whole society will get infections over time like in the orange curve. Unfortunately, this is not true. The people from the blue curve will infect the people from the green curve much earlier than the people of the green curve would do if they were alone. Assuming that the careful ones of the society will not infect so many people but were also infected equally by the careless people, one will get two coupled differential equations:

$$
\dot{n}_{1}+\dot{n}_{2}=\frac{n_{1}}{\tau_{1}} \cdot\left(1-2 n_{1}\right)+\frac{n_{2}}{\tau_{2}} \cdot\left(1-2 n_{2}\right) \quad \dot{n}_{1}=\dot{n}_{2}
$$

The indices 1 and 2 refer to the two halves of the society. $n(t)=n_{1}(t)+n_{2}(t)$ is the entire society. The first equation in Equation (5) states the infection rate of the entire population $n_{1}+n_{2}$. Both population groups have the limit $1 / 2$. The second equation states that both halves of population are infected equally. Of course one can modify this. One can also generalize Equation (5) so that the two parts of the population are not equal in size. Equation (6) is valid when only ten percent are in group 1 and ninety percent are in group 2 :

$$
\dot{n}_{1}+\dot{n}_{2}=\frac{n_{1}}{\tau_{1}} \cdot\left(1-10 n_{1}\right)+\frac{n_{2}}{\tau_{2}} \cdot\left(1-\frac{10}{9} n_{2}\right) \quad 9 \dot{n}_{1}=\dot{n}_{2}
$$

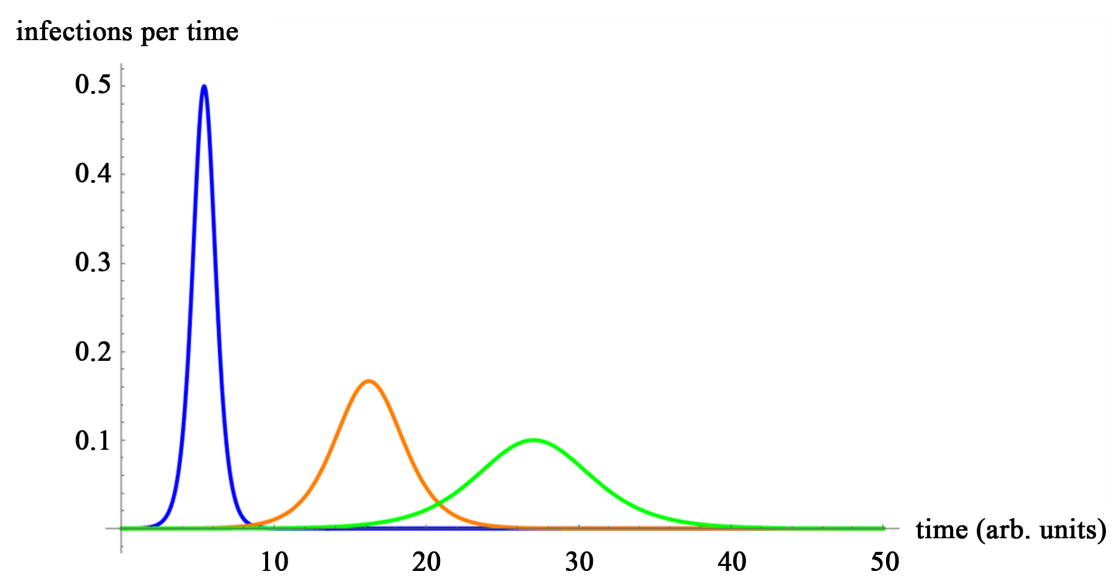

Figure 2. Plot of Equation (4) for $\tau=0.5,1.5$, and 2.5 . 


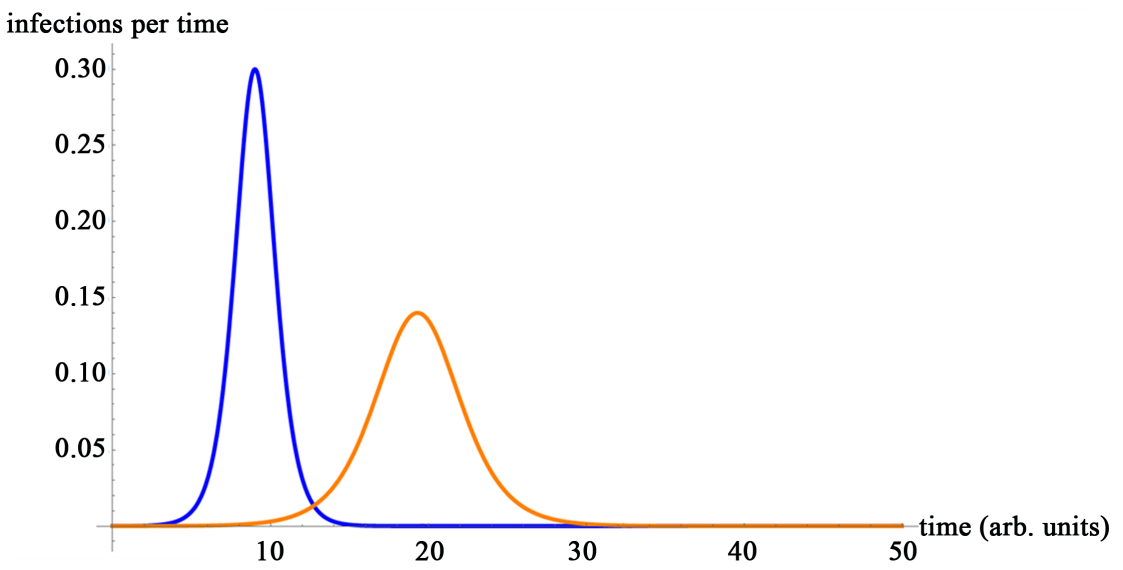

Figure 3. Total infections $n(t)$ with $\tau_{1}=0.5$ and $\tau_{2}=2.5$. Blue curve from Equation (5), $n_{01}=n_{02}=10^{-5}$, and orange curve from Equation (6), $n_{01}=2 \times 10^{-6} n_{02}=18 \times 10^{-6}$.

The factors in Equation (6) are chosen so that the ratio of $n_{1}$ to $n_{2}$ is 1 to 9 . Equations (5) or (6) are integrable. But the result cannot be written down in a closed form. We have therefore chosen to solve Equations (5) and (6) numerically. In Figure 3, we have plotted the solution of Equation (5) in the blue curve. The result is only slightly different from the blue curve in Figure 2. If half of the population behaves like in the blue curve of Figure 2 and half like the green curve, it is only slightly better compared to the situation where all people behave like the blue curve in Figure 2. The effort of the very careful ones is (almost) wasted. Even if ninety percent are behaving very carefully (orange curve in Figure 3), we are only slightly better off than the orange curve in Figure 2.

\section{Conclusions and Further Work}

We have shown that the bad behavior of one half cannot be compensated by the very good behavior of the other half in order to reach the goal of spreading cases of a disease over a long period. We have a situation where a very broad majority must take appropriate measures as otherwise measures become effectless. As we see from the orange curve in Figure 3, ten percent can tremendously change the outcome for everybody though ninety percent show a perfect behavior. It is therefore not enough that politicians look to a broad majority. They have to scrutinize the ten percent ignoring the pandemic.

A final discussion with real numbers is only possible after the pandemic. But even today the findings of this letter may (partly) explain why European countries are hit so differently. The culture of obeying rules is very different in different countries.

As an extension to this work one may use a distribution of behavior instead of just two values for $\tau$. However, this leads to extremely complicated mathematics, but most likely does not change results much.

Having non-linear differential equations one may also look for chaos, see e.g. Schuster, 1984. Equations (5) and (6) will (most likely) never show chaos in 
themselves. However, changing the model at a certain point may have dramatic effects. In Equations (5) or (6) it would mean changing the $\tau$ at a certain point in time. Politically speaking it means taking a certain measure such as a curfew earlier or later. In doing so chaos effects are likely. Such chaos effects are more like "the drop of honey effect" as defined in Filipe \& Ferreira, 2013 (see also Filipe et al., 2010) in contrast to chaos effects like in the weather forecast.

Furthermore, people often speak of panic in the current Covid-19 pandemic. A panic is an abrupt change of behavior. In our model, it is a (discontinuous) change in $\tau$ or the ratio of $n_{1}$ to $n_{2}$. In doing so chaos effects are very likely. This might be a good quantification of the above mentioned "drop of honey effect."

\section{Conflicts of Interest}

The authors declare no conflicts of interest regarding the publication of this paper.

\section{References}

Black, F., \& Scholes, M. (1973). The Pricing of Options and Corporate Liabilities. Journal of Political Economy, 81, 637-654. https://doi.org/10.1086/260062

Bronshtein, I. N., Semenddyayev, K. A., Musiol, G., \& Muehlig, H. (2007). Handbook of Mathematics (5th English Edition). Berlin Heidelberg: Springer.

Filipe, J. A., \& Ferreira, M. A. M. (2013). Social and Political Events and Chaos Theory: The "Drop of Honey Effect". Emerging Issues in the Natural and Applied Sciences, 3, 126-137.

Filipe, J. A., Ferreira, M. A. M., Coelho, M., \& Pedro, M. I. (2010). Chaos, Anti-Chaos and Resources: Dealing with Complexity. Journal of Applied Mathematics, 3, 83-90.

Grabinski, M., \& Klinkova, G. (2019). Wrong Use of Averages Implies Wrong Results from Many Heuristic Models. Applied Mathematics, 10, 605-618. https://doi.org/10.4236/am.2019.107043

Schuster, H. G. (1984). Deterministic Chaos. Physik Verlag, Weinheim.

The Economist (2020). Briefing Covid19, Feb. 29, 2020, 15. https://www.economist.com/briefing/2020/02/29/covid-19-is-now-in-50-countries-and -things-will-get-worse

Weiber, R. (1993) Chaos: Das Ende der klassischen Diffusionsmodellierung? Marketing ZFP, 1, 35-46. https://doi.org/10.15358/0344-1369-1993-1-35 